\title{
Chlamydia trachomatis and Neisseria gonorrhoea Co- Infection Among Patients Attending a Teaching Hospital in Nairobi County: A Retrospective Study
}

\author{
Festus Mulakolia \\ Kenyalta university-Nairobi, Kenya \\ * Correspondence to Mr. Festus Mulakoli ( mulakolifesto@gmail.com)
}

\begin{abstract}
Background: Chlamydia trachomatis and Neisseria gonorrhoea are microbes that have been associated with urethritis in both male and female genders, which often may lead to complicated conditions such as pelvic inflammatory disease (PID) and infertility globally among others health complications. In Kenya and other developing countries, sexually transmitted infections associated with Chlamydia trachomatis and Neisseria gonorrhoea still pose a challenge in public health.
\end{abstract}

Methods: A retrospective study was conducted by reviewing laboratory data from Jan 2018 to Dec 2018 to estimate the prevalence of $C$ trachomatis and $N$ gonorrhoea coinfections in patients attending a tertiary institution and its satellite clinics spread across the country. A total of 1228 patient's data aged 3-69 years was reviewed; with age, gender and Chlamydia trachomatis and Neisseria gonorrhoea status being analyzed.

Results: A total of 1228 patients who visited the hospital in 2018 had their urine samples being tested for Chlamydia trachomatis and Neisseria gonorrhoea by use of a PCR technique. Majority of the patients were males (63.7\%). The patients who tested for Chlamydia trachomatis and Neisseria gonorrhoea had an average age of 34 years (range: 3-69 years). Of those $1.4 \%$ tested positive for both Chlamydia trachomatis and Neisseria gonorrhoea infections, and males were more infected than females (1.1\% vs 0.3$)$.

From the information gathered during the study period, the proportion of patients with Chlamydia trachomatis infection was (16.1 \%) $(95 \% \mathrm{Cl} 9.5,17.9)$, and with N. gonorrhoea infection was 5.4\%. Coinfection was highest among sexually active group that is those aged between 21 years to 40 years.

Conclusion: The prevalence of $C$. trachomatis is significantly high among male patients. We recommend the implement a molecular screening for Chlamydia trachomatis and Neisseria gonorrhoea to identifying asymptomatic female cases. This study further provides evidence on the importance of contact tracing in the management of Chlamydia trachomatis and other STIs. There is an urgent need for studies designed to investigate the prevalence and risk factors of Chlamydia trachomatis and Neisseria gonorrhoea among female patients who are majorly asymptomatic in Kenya.

\section{INTRODUCTION}

Teisseria gonorrhoea and Chlamydia trachomatis 1 are microbes that have been associated with urethritis in both males and females, which often may lead to complicated conditions such as pelvic inflammatory disease (PID) and infertility globally. ${ }^{1}$ Therefore, early detection and clinical management are important in eliminating these complications. Gonococcal urethritis is caused by a gram-negative diplococci bacterium commonly associated with sexually transmitted infections (STI) in males and female patients that manifests either symptomatic or asymptomatic. ${ }^{2}$ Early gonococcal infection in women is always asymptomatic which may further spreads to the upper part of the genital tract causing salpingitis. According to WHO, the estimated global prevalence of Chlamydia trachomatis 
and gonorrhoea stands at $4.2 \%$ and $0.8 \%$ respectively. In 2014 for instance over 1 million sexually transmitted disease (STD) cases were thought to be reported per day. ${ }^{3}$ A study by Kularatne et al ${ }^{4}$ in South Africa estimated that the prevalence of $N$. gonorrhoea and Chlamydia trachomatis was at $6.6 \%$ and $14.7 \%$ among female patients respectively. The male had a prevalence of $3.5 \%$ and $6.0 \%$ for $N$. gonorrhoea and Chlamydia trachomatis respectively in 2017 among adult patients aged between $15-49$ years. ${ }^{4}$ Screening for STIs among patients is, therefore, an important way of identifying asymptomatic individuals earlier enough before any complications.,5 Indeed, studies have shown that earlier detection of STIs minimizes their sequelae effect in the affected individuals. ${ }^{7,8}$

Information currently available from surveillance data on most common STIs among patients in Kenya has been largely from studies done among high-risk groups $^{9,10}$ and special categories like expectant mothers. ${ }^{11}$ There is however missing knowledge about the point prevalence of STIs among patients in the general population. This is because the surveillance of STIs, although recommended ${ }^{12,13,14}$, has not been well implemented; with the major focus in Kenya in the last $10-15$ years being on HIV, Tb, and Malaria. This is further complicated by the fact that surveillance guidelines exist for the screening of general patients for curable STIs and utilization of laboratory data to monitor disease patterns in the communities. Further, studies have demonstrated that a large number of infected people with no reported clinical symptoms act as a disease reservoir and act as a source of infection to susceptible groups if not detected on time. ${ }^{6}$ Therefore, this study aimed to determine the point prevalence for C. trachomatis, $N$. gonorrhoea coinfection in the general patient population attending a tertiary teaching institution. Knowledge generated from this study is important for clinicians and health policy makers to aid them in coming up with comprehensive diagnostic guidelines.

\section{METHODS}

This was a retrospective descriptive observational study conducted at tertiary teaching and referral hospital in Nairobi, Kenya by reviewing laboratory data between January to December 2018. The hospital offers comprehensive laboratory services, with its main laboratory opened for 24 hrs. The laboratory offers molecular diagnostic services for the detection of infectious disease including Chlamydia trachomatis and Neisseria gonorrhoea. The study reviewed all patient's laboratory data that included the patient's age, gender and laboratory molecular results for Chlamydia trachomatis and Neisseria gonorrhoea. The study inclusion criteria were all the patients served during the selected review period. Patients with missing a laboratory report were excluded from the study. Data collected were entered into Microsoft Excel and statistically analyzed using IBM SPSS Statistics for Windows, Version 20.0. Armonk, NY: IBM Corp. Chi-square and Fisher's exact tests were used to determining univariate social demographic factors associations of $C$. trachomatis and $N$. gonorrhoea co-infection. A p-value of $<0.05$ was considered to be statistically significant.

\section{RESULTS}

A total of 1228 patients had their urine samples tested for Chlamydia trachomatis and Neisseria gonorrhoea by use of a PCR molecular technique in 2018. The patients had an average age of 34 years with an age range between 3-69 years as shown in Table 1. The patients that were tested for Chlamydia trachomatis and Neisseria gonorrhoea were aged between 31-40 years $(6.9 \%, 2.8 \%)$, followed by those between $21-30$ $(6.0 \%, 1.4 \%)$. The majority of the patients were men $781(63.6 \%)$ and $447(36.4 \%)$ were female as summarized in Table 2. 
TABLE 1: Calculated mean age and distribution of the review participants

\begin{tabular}{lc}
\hline & Frequency \\
\hline Mean Age & 34 \\
SD & 9 \\
Median & 33 \\
Max & 69 \\
Min & 3 \\
Age groups & \\
Below 20 & 52 \\
$21-30$ & 421 \\
$31-40$ & 502 \\
$41-50$ & 187 \\
Above 50 & 66 \\
\hline
\end{tabular}

TABLE 2: Distribution of $\mathrm{C}$ trachomatis and $\mathrm{N}$ gonorrhea infection across age groups and gender.

\begin{tabular}{|c|c|c|c|c|c|}
\hline & \multicolumn{4}{|c|}{ C trachomatis $N$ gonorrhea } & \multirow[t]{2}{*}{$n(\%)$} \\
\hline & $\begin{array}{c}\text { CTNG-Negative } n \\
(\%)\end{array}$ & $\begin{array}{c}\text { CT-Positive } n \\
(\%)\end{array}$ & $\begin{array}{c}\text { NG-Positive } n \\
(\%)\end{array}$ & $\begin{array}{c}\text { CTNG- } \\
\text { Positive } \\
\text { n }(\%)\end{array}$ & \\
\hline $\begin{array}{l}\text { Under } \\
20\end{array}$ & $39(3.2 \%)$ & $9(0.7 \%)$ & $4(0.3 \%)$ & $1(0.08 \%)$ & $53(4.3 \%)$ \\
\hline $21-30$ & $321(26.1 \%)$ & $74(6.0 \%)$ & $17(1.4 \%)$ & $9(0.7 \%)$ & $421(34.3 \%)$ \\
\hline $31-40$ & $378(30.8 \%)$ & $85(6.9 \%)$ & $34(2.8 \%)$ & $7(0.6 \%)$ & $504(41.0 \%)$ \\
\hline $41-50$ & $159(12.9)$ & $20(1.6 \%)$ & $9(0.7 \%)$ & $0(0.0 \%)$ & $188(15.3 \%)$ \\
\hline $\begin{array}{l}\text { above } \\
50\end{array}$ & $50(4.1 \%)$ & $10(0.8 \%)$ & $2(0.2 \%)$ & $0(0.0 \%)$ & $62(5.0 \%)$ \\
\hline Male & $559(45.5 \%)$ & $148(12.1 \%)$ & $61(5.0 \%)$ & $13(1.1 \%)$ & $781(63.6 \%)$ \\
\hline Female & $388(31.6 \%)$ & $50(4.1 \%)$ & $5(0.4 \%)$ & $4(0.3 \%)$ & $447(36.4 \%)$ \\
\hline
\end{tabular}


From the information gathered during the study, Chlamydia trachomatis had the highest prevalence of $16 \%(198 / 1228)(95 \%$ CI $9.5,17.9)$, and $N$ gonorrhoea at 66/1228 (5\%) as shown in Figure 1. A co-infection of Chlamydia trachomatis and $N$ gonorrhoea was found in $17 / 1228(2 \%)$ of the sampled data. Of all the patients 947 (77\%) were found to have no Chlamydia trachomatis and Neisseria gonorrhoea infection during the review period Figure 1 .

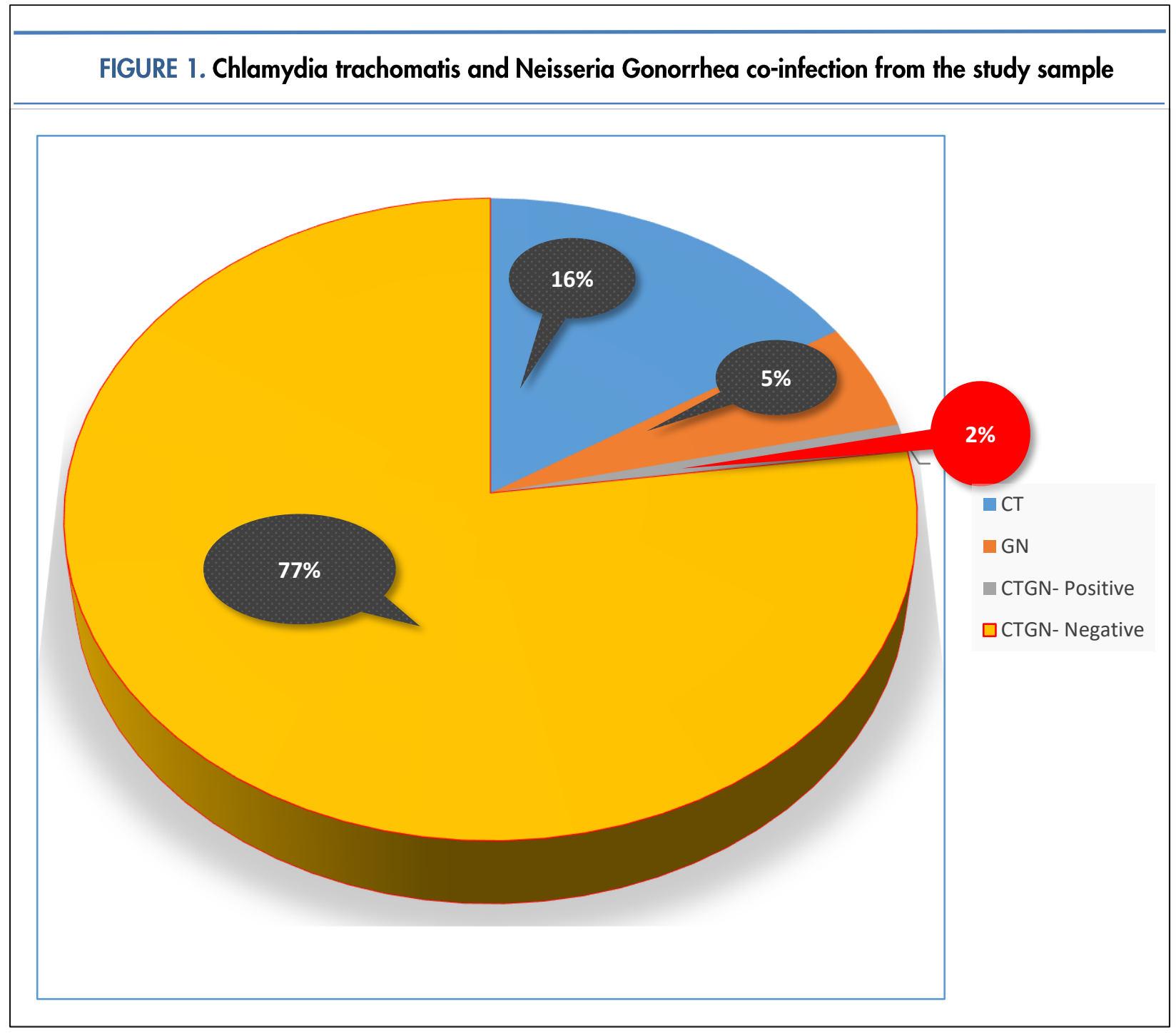

Higher frequency of Chlamydia trachomatis and $N$ gonorrhoea were observed among patients aged 31-40 years which was at $6.9 \%$ and $2.8 \%$ respectively and lowest $(0.7 \%, 0.3 \%)$ among patients aged below 20 years respectively. The male patients exhibited the highest prevalence of $\mathrm{n}=148 / 198(74.7 \%)$ as presented in Figure 1. Coinfection was highest at 9/17 (52.2\%) among patients aged between 21-30 years and among the male patients at $13 / 17(76.5 \%)$. Least prevalence was seen in the age groups above 40 years Table 2 . 


\section{DISCUSSION}

This study estimated point prevalence of $C$. trachomatis and N. gonorrhoea co-infection among patients whose urine sample were tested by a PCR technique. The point prevalence of $C$. trachomatis and $N$. gonorrhoea co-infection as reported by this study stood at $1.4 \%$ (in the year 2018. With the highest prevalence being seen in the male patients aged between 31-40 yrs. with a pvalue of 0.31 . However, patients above 40 years had the least prevalence was recorded. These study findings were comparable to $2.8 \%$ reported in previously published studies among female patients at a US juvenile centre and with a lower prevalence as compared to $4.6 \%$ reported among men how to have sex with men. ${ }^{15,16}$

A higher Chlamydia trachomatis prevalence of $16 \%$ was also reported in this study among patients aged 3041 years. Similar studies have found a prevalence of between 6 and $16 \%$ among female patients attending family planning clinics in Nairobi- Kenya. ${ }^{17,10,18}$ What we can learn from this is that the prevalence of $C$. trachomatis is of great concern among the male patients and individuals in their mid-years. This high numbers could be attributed to the symptomatic nature of $C$ trachomatis among the male gender and increased sexual activities among individuals in their mid-years. Therefore, introducing a molecular screening procedure for routine screening of STIs will help in reducing the burden of the disease. ${ }^{7}$

However, a point prevalence of $5 \%$ for $N$. gonorrhoea is less than what is seen in initially published work by Marx et $a^{19}$ from a similar setting who found an $N$. gonorrhoea prevalence of $6 \%$ among HIV-1 infected pregnant patients in Nairobi; in another study by Fonck et al..$^{20}$ he found a prevalence of $6 \%$ for Chlamydia trachomatis and $4 \%$ for Neisseria gonorrhoea among patients with complaints of vaginal discharge attending a sexually transmitted diseases (STD) referral clinic in Nairobi. While Daly et al. ${ }^{21}$ found a Neisseria gonorrhoea prevalence of $3.2 \%$ among patients seeking treatment in Nairobi which is lower as compared to what was found in this study.

It was not clear why this study recorded a lower prevalence of Chlamydia trachomatis and gonorrhoea among female patients as most studies have shown that a higher prevalence among female patients. However, the female patients were the minority in our study as compared to male patients. The higher Neisseria gonorrhoea and Chlamydia trachomatis prevalence could be attributed to a good precision of the molecular method as compared to traditional culture methods..$^{22,23}$
All the patients that turned out to be positive Chlamydia trachomatis and $N$ gonorrhoea were aged between the 21-50 and the male patients being the majority. These findings, therefore, suggest that the male patients were symptomatic as compared to the female gender. These further depict the importance of contact tracing, testing, and treatment to reduce reinfection. ${ }^{24}$

The high proportion of symptomatic patients who tested positive for $C$. trachomatis that is in agreement with other findings and further emphasizes the importance of molecular techniques in the diagnosis and management of curable STIs. ${ }^{24}$ However, due to the high cost of molecular techniques and poorly equipped laboratory in most of the developing countries, the WHO guidelines recommend a syndromic approach in the management of STIs. ${ }^{25}$ Syndromic management consists of a group of symptoms and easily recognized signs that are identified before treatment. ${ }^{26}$

\section{CONCLUSION}

The prevalence of $C$. trachomatis is significantly high among male patients. We recommend the implement a molecular screening for Chlamydia trachomatis and Neisseria gonorrhoea to identifying asymptomatic female cases. This study further provides evidence on the importance of contact tracing in the management of Chlamydia trachomatis and other STIs. There is an urgent need for studies designed to investigate the prevalence and risk factors of Chlamydia trachomatis and Neisseria gonorrhoea among female patients who are majorly asymptomatic in Kenya.

\section{Ethical consideration}

Ethics approval was not sorted for this retrospectively obtained and anonymized data non-interventional study.

\section{Acknowledgement: N/A}

Financial support

No financial support offered

\section{Conflict of interest}

There was no conflict of interest 


\section{REFERENCE}

1. Jennings LK, Krywko DM. Pelvic Inflammatory Disease (PID).; 2019.

2. Stevens JS, Criss AK. Pathogenesis of Neisseria gonorrhoeae in the female reproductive tract: Neutrophilic host response, sustained infection, and clinical sequelae. Curr Opin Hematol. 2018;25(1):13-21. doi:10.1097/MOH.0000000000000394

3. WHO. WHO | Global incidence and prevalence of selected curable sexually transmitted infections - 2008. WHO. 2014.

4. Kularatne RS, Niit R, Rowley J, et al. Adult gonorrhoea, Chlamydia trachomatis and syphilis prevalence, incidence, treatment and syndromic case reporting in South Africa: Estimates using the Spectrum-STI model, 1990-2017. PazBailey G, ed. PLoS One. 2018;13(10):e0205863. doi: 10.1371 /journal.pone. 0205863

5. Taylor MM, Frasure-Williams J, Burnett P, Park IU. Interventions to improve sexually transmitted disease screening in clinic-based settings. Sex Transm Dis. 2016;43(2): S28-S41. doi:10.1097/OLQ.0000000000000294

6. Farley TA, Cohen DA, Elkins W. Asymptomatic sexually transmitted diseases: The case for screening. Prev Med (Baltim). 2003. doi:10.1016/S0091-7435(02)00058-0

7. Pillay J, Moore A, Rahman P, et al. Screening for Chlamydia trachomatis and/or gonorrhoea in primary health care: Protocol for a systematic review. Syst Rev. 2018;7(1). doi:10.1186/s13643-018-0904-5

8. Mary K, Turner E. Mathematical models of gonorrhoea and Chlamydia trachomatis: biology, behaviour and interventions.

9. Masese L, Baeten JM, Richardson BA, et al. Incidence and Correlates of Chlamydia trachomatis trachomatis Infection in a High-Risk Cohort of Kenyan Women. Sex Transm Dis. 2013;40(3):221-225. doi:10.1097/OLQ.0b013e318272fe45

10. Maina AN, Kimani J, Anzala O. Prevalence and risk factors of three curable sexually transmitted infections among women in Nairobi, Kenya. BMC Res Notes. 2016;9:193. doi:10.1186/s13104-016-1990-X

11. Masha SC, Wahome E, Vaneechoutte M, Cools P, Crucitti T, Sanders EJ. High prevalence of curable sexually transmitted infections among pregnant women in a rural county hospital in Kilifi, Kenya. Fredricks DN, ed. PLoS One. 2017;12(3):e0175166. doi:10.1371/journal.pone.0175166

12. WHO. Global incidence and prevalence of selected curable sexually transmitted infections. WHO Rep. 2008.

13. Ministry of Health Kenya. National Adolescent Sexual and Reproductive Health Policy, 2015.; 2015. doi:10.1017/CBO9781107415324.004

14. WHO. Report on Global Sexually Transmitted Infection Surveillance 2013.;
doi:10.1080/10288457.1997.10756086
15. Kahn RH, Mosure DJ, Blank S, et al. Chlamydia trachomatis trachomatis and Neisseria gonorrhoeae Prevalence and Coinfection in Adolescents Entering Selected US Juvenile Detention Centers, 1997-2002 the jail std prevalence monitoring project. Am Sex Transm Dis Assoc. 2005;32(4):255-259.

doi:10.1097/01.olq.0000158496.00315.04

16. Sanders EJ, Thiong'. UvA-DARE (Digital Academic Repository) Link to publication Citation for published version (APA). Sex Transm Infect. 2010;86:440-441. doi:10.1136/sti.2010.043224

17. Kohli R, Konya WP, Obura T, Stones W, Revathi G. Prevalence of genital Chlamydia trachomatis infection in urban women of reproductive age, Nairobi, Kenya. BMC Res Notes. 2013;6(1). doi:10.1186/1756-0500-6-44

18. Masese L, Baeten JM, Richardson BA, et al. Incidence and correlates of Chlamydia trachomatis trachomatis infection in a high-risk cohort of Kenyan women. Sex Transm Dis. 2013;40(3):221-225. doi:10.1097/OLQ.0b013e318272fe45

19. Marx G, John-Stewart G, Bosire R, Wamalwa D, Otieno P, Farquhar C. Diagnosis of sexually transmitted infections and microbesl vaginosis among HIV-1-infected pregnant women in Nairobi. Int $J$ STD AIDS. 2010;21(8):549-552. doi:10.1258/ijsa.2010.010005

20. K. F, N. K, P. K, et al. Pattern of sexually transmitted diseases and risk factors among women attending an STD referral clinic in Nairobi, Kenya. Sex Transm Dis. 2000;27(7):417423.

21. Daly CC, Maggwa N, Mati JK, et al. Risk factors for gonorrhoea, syphilis, and trichomonas infections among women attending family planning clinics in Nairobi, Kenya. Genitourin Med. 1994;70(3):155-161. doi:10.1136/sti.70.3.155

22. Fuller SS, Chow SC, Nori A V, et al. Diagnostic accuracy of a prototype rapid Chlamydia trachomatis and gonorrhoea recombinase polymerase amplification assay : a multicentre cross-sectional preclinical evaluation *. Clin Microbiol Infect. 2019;25(3):380.e1-380.e7. doi:10.1016/j.cmi.2018.06.003

23. Parcell BJ, Ratnayake L, Kaminski G, Olver WJ, Yirrell DL. Value of repeat testing using Cepheid GeneXpert CT/NG for indeterminate PCR results when diagnosing Chlamydia trachomatis trachomatis and Neisseria gonorrhoeae. Int $\mathrm{J}$ STD AIDS. 2015;26(1):65-67. doi:10.1177/0956462414531938

24. Hui BB, Wilson DP, Ward JS, et al. The potential impact of new generation molecular point-of-care tests on gonorrhoea and Chlamydia trachomatis in a setting of high endemic prevalence. Sex Health. 2013. doi:10.1071/SH13026

25. WHO. Guidelines for the Management of Sexually Transmitted Infections.; 2001.

26. Health NRC (US) P on R, Tsui AO, Wasserheit JN, Haaga JG. WHO Recommendations for Treatment of STD-Associated Syndromes. 1997. 
Peer Reviewed

Competing Interests: None declared.

Received: 6/9/2020; Accepted: 2/22/2021

Cite this article as: Mulakoli F. Chlamydia trachomatis and Neisseria gonorrhoea Co-Infection Among Patients Attending a Teaching Hospital in Nairobi County: A Retrospective Study

. E Afr Sci. 2021;3(1):51-57. http://doi.org/10.24248/EASci-D20-00008

(1) Mulakoli F. This is an open-access a rticle distributed under the terms of the Creative Commons Attribution License, which permits unrestricted use, distribution, and reproduction in any medium, provided the original author and source are properly cited. To view a copy of the license, visit http://creativecommons.org/licens- es/by/4.0/. When linking to this article, please use the following permanent link: http://doi.org/10.24248/EASCi-D-20-00008 